\title{
Análise hidrogeomorfométrica da microbacia do rio Mutum: informações para auxi- liar o manejo de recursos hídricos na Amazônia Ocidental
}

\author{
Hydrogeomorphometric analysis of the Mutum river microbasin: information to assist the manage- \\ ment of water resources in the Western Amazon
}

Análisis hidrogeomorfométrica de la microcuenca del río Mutum: información para ayudar a la gestión de los recursos hídricos en la Amazonía occidental

Recebido: 28/01/2021 | Revisado: 02/02/2021 | Aceito: 05/02/2021 | Publicado: 11/02/2021

Tiago Way Serrão de Souza

ORCID: https://orcid.org/0000-0003-3787-5014

Universidade Federal do Amazonas, Brasil

E-mail: wayserrao@gmail.com

Marcelo Medeiros de Lima

ORCID: https://orcid.org/0000-0002-3347-8815

Universidade Federal do Amazonas, Brasil

E-mail: delima.job@gmail.com

Jhade Goudinho Saraiva

ORCID: https://orcid.org/0000-0003-2491-1602

Universidade Federal do Amazonas, Brasil

E-mail: jgsjhade@gmail.com

Fabrício Matheus Pimenta Pacheco

ORCID: https://orcid.org/0000-0002-4705-7034

Universidade Federal do Amazonas, Brasil

E-mail: fabriciomatheus95@gmail.com

Miquel Victor Batista Donegá

ORCID: https://orcid.org/0000-0003-4904-5552

Universidade Federal do Amazonas, Brasil E-mail: miquel@ufam.edu.br

Mylena Rego Panza

ORCID: https://orcid.org/0000-0002-3756-8017 Universidade Federal do Amazonas, Brasil E-mail: panzamylena@gmail.com

Wanderson Cleiton Schmidt Cavalheiro

ORCID: https://orcid.org/0000-0003-1356-8511 Cavalheiro Engenharia Rural e Empresarial LTDA, Brasil E-mail: engflo.ro@gmail.com

Jhony Vendruscolo

ORCID: https://orcid.org/0000-0003-3043-0581

Universidade Federal do Amazonas, Brasil

E-mail: jhonyvendruscolo@gmail.com

\begin{abstract}
Resumo
O desenvolvimento sustentável de sistemas agropecuários tem como base o planejamento e a gestão dos recursos naturais, logo, é essencial conhecer as características da paisagem. Em face ao exposto, o presente trabalho tem como objetivo fornecer informações sobre as características hidrogeomorfométricas da microbacia do rio Mutum. A análise da paisagem foi realizada com os softwares Google Earth, QGIS e TrackMaker Free, imagens altimétricas dos satélites Alos, e dados da literatura. A microbacia tem área de $8,64 \mathrm{~km}^{2}$, perímetro de $15,26 \mathrm{~km}$, formata alongada e baixa a média suscetibilidade a inundações (ponto de vista geométrico), altitudes de 228 a $322 \mathrm{~m}$, predominância de relevos suave ondulado $(50,81 \%)$ e ondulado $(30,67 \%), 92,93 \%$ de sua área com baixa propensão a influência a propagação de incêndios e 92,94\% apta à extremamente apta a mecanização agrícola, rede de drenagem de $17,30 \mathrm{~km}$ com padrão dendrítico de $4^{\mathrm{a}}$ ordem, densidades de nascentes média, densidade de drenagem média, coeficiente de manutenção de $499,4 \mathrm{~m}^{2} \mathrm{~m}^{-1}$, canal principal reto e baixo tempo de concentração. A microbacia tem potencial para o desenvolvimento agropecuário, no entanto, a adoção de práticas de manejo conservacionista do solo é necessário para mitigar os impactos desta atividade no meio ambiente. É recomendado o estudo da dinâmica temporal e espacial da cobertura do solo, para analisar a qualidade e a quantidade de vegetação nativa na microbacia, e assim, delimitar áreas prioritárias para a manutenção e/ou recuperação dessa vegetação.
\end{abstract}

Palavras-chave: Geotecnologias; Características da paisagem; Análise ambiental; Manejo dos recursos naturais. 


\begin{abstract}
Sustainable development of agricultural systems is based on the planning and management of natural resources, so it is essential to know the landscape features. In view of the above, the present work aims to provide information on the hydrogeomorphometric characteristics of the Mutum river microbasin. The landscape analysis was performed with the Google Earth, QGIS and TrackMaker Free software, altimetric images from the Alos satellites, and data from the literature. The microbasin has an area of $8.64 \mathrm{~km}^{2}$, a perimeter of $15.26 \mathrm{~km}$, elongated formations and low to medium susceptibility to flooding (geometric point of view), altitudes from 228 to $322 \mathrm{~m}$, predominance of smooth wavy $(50.81 \%)$ and wavy reliefs (30.67\%), $92.93 \%$ of its area with low propensity to influence the spread of fires and $92.94 \%$ suitable for apt agricultural mechanization, drainage network of $17.30 \mathrm{~km}$ with dendritic pattern 4th order, average spring densities, average drainage density, maintenance coefficient of $499.4 \mathrm{~m}^{2} \mathrm{~m}^{-1}$, straight main channel and low concentration time. The microbasin has the potential for agricultural development, however, the adoption of conservationist soil management practices is necessary to mitigate the impacts of this activity on the environment. We recommend a study on the spatial and temporal dynamics of land cover, to analyze the quality and quantity of native vegetation in the microbasin, and thus, delimit priority areas for the maintenance and/or recovery of this vegetation.
\end{abstract}

Keywords: Geotechnologies; Landscape characteristics; Environmental analysis; Management of natural resources.

\title{
Resumen
}

El desarrollo sostenible de los sistemas agrícolas se basa en la planificación y gestión de los recursos naturales, por lo que es fundamental conocer las características del paisaje. En vista de lo anterior, el presente trabajo tiene como objetivo proporcionar información sobre las características hidrogeomorfométricas de la microcuenca del río Mutum. El análisis del paisaje se realizó con el software Google Earth, QGIS y TrackMaker Free, imágenes altimétricas de los satélites Alos y datos de la literatura. La microcuenca tiene un área de $8,64 \mathrm{~km}^{2}$, un perímetro de $15,26 \mathrm{~km}$, forma alargada, baja a media susceptibilidad a inundaciones (punto de vista geométrico), altitudes de 228 a $322 \mathrm{~m}$, predominio de relieves suave ondulado $(50,81 \%)$ y ondulado $(30,67 \%), 92,93 \%$ del área con baja propensión a influir en la propagación de incendios, 92,94\% del área considerada apta para la mecanización agrícola, red de drenaje de 17,30 km con patrón dendrítico de $4^{\circ}$ orden, densidades medias de manantial, densidad media de drenaje, coeficiente de mantenimiento de $499,4 \mathrm{~m}^{2} \mathrm{~m}^{-1}$, canal principal recto y tiempo de concentración bajo. La microcuenca tiene potencial para el desarrollo agrícola, sin embargo, la adopción de prácticas conservacionistas de manejo de suelos es necesaria para mitigar los impactos de esta actividad en el medio ambiente. Se recomienda estudiar la dinámica temporal y espacial de la cobertura terrestre, para analizar la calidad y cantidad de vegetación nativa en la microcuenca, y así delimitar áreas prioritarias para el mantenimiento y/o recuperación de esta vegetación.

Palabras clave: Geotecnologías; Características del paisaje; Análisis ambiental; Manejo de recursos naturales.

\section{Introdução}

A microbacia do rio Mutum está inserida na bacia do rio Guaporé e sub-bacia do rio Vermelho, e tem grande relevância ambiental, visto que o rio Guaporé forma um corredor ecológico que conecta as regiões biogeográficas da Amazônia e Pantanal (Silva, et al, 2015), e econômica, por abranger 20 estabelecimentos agropecuários privados (INCRA, 2019). Neste contexto, constata-se a necessidade de obter informações da paisagem para elaborar estratégias de uso e conservação dos recursos naturais, e assim, garantir o desenvolvimento sustentável da região.

A microbacia é a unidade ideal de gestão dos recursos naturais na região amazônica, por permitir um planejamento mais eficiente devido a aquisição de dados detalhados da paisagem, em relação a bacias e sub-bacias (Cavalheiro \& Vendruscolo, 2019). Esta unidade de gestão é considerada ideal por possibilitar o uso integrado dos recursos hídricos (Brasil, 1997), ao captar a água da chuva, abastecer o lençol freático, disponibilizar água ao longo do ano, por meio de nascentes e uma rede de drenagem (Tucci, 1993), e refletir o impacto das ações antrópicas na qualidade e disponibilidade deste recurso natural (Tambosi, et al., 2015).

Para elaborar um planejamento econômico e ambiental eficiente para a microbacia é necessário analisar as características da paisagem. Estas características estão associadas às informações hidrogeomorfométricas, que denotam as potencialidades econômicas e limitações dos recursos naturais, auxiliando a seleção de espécies de cultivo agrícolas e florestais, seleção de práticas de manejo do solo para mitigar o impacto das atividades antrópicas sobre os recursos naturais, e delimitação de áreas prioritárias para a manutenção de vegetação nativa (Moreto, et al., 2019; Panza, et al., 2020; Vendruscolo, et al., 2021). As informações da paisagem podem ser obtidas em tempo hábil e com baixo custo financeiro por meio de geotecnologias, que 
também facilitam a análise ambiental (Soares, et al., 2019a).

Neste contexto, o trabalho tem como objetivo disponibilizar as informações das características da paisagem na microbacia do rio Mutum, para auxiliar no desenvolvimento sustentável dos estabelecimentos agropecuários privados e manter a qualidade dos ecossistemas naturais da região.

\section{Metodologia}

\subsection{Localização e caracterização da área de estudo}

A microbacia do rio Mutum está inserida nos municípios de Cabixi (77,89\%) e Colorado D’Oeste (22,11\%), no estado de Rondônia (Figura 1). Esta localidade tem clima classificado como Monção, com temperaturas de 24 a $26^{\circ} \mathrm{C}$ (Alvares, et al., 2014), precipitações anuais de $1.728,9$ a $1.843,7 \mathrm{~mm}$, sendo mais elevadas durante os meses de novembro a março e mais escassas durante os meses de junho a agosto (Franca, 2015), e solos classificados com Argissolo Vemelho eutrófico (98,32\%) e Gleissolo (1,68\%) (SEDAM, 2002).

Figura 1. Localização da microbacia do rio Mutum, Amazônia Ocidental, Brasil.

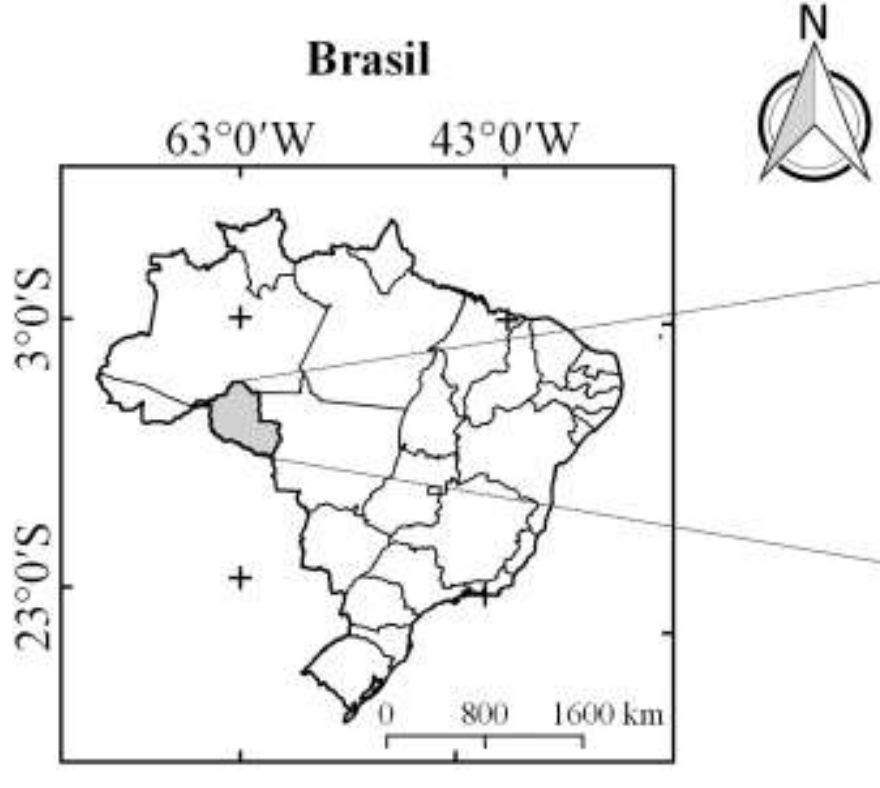

Microbacia do rio Mutum

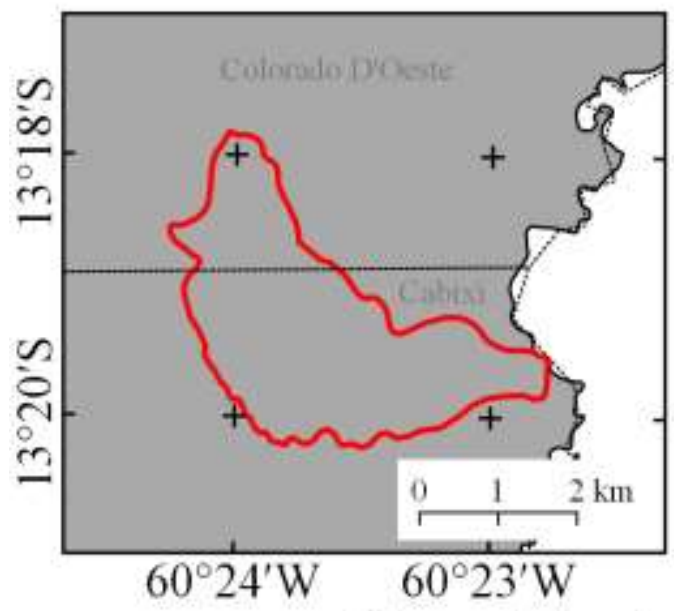

Sub-bacia do rio Vermelho

Sistema de Coordenadas Geográficas - Datum WGS 84

Fonte: Autores. 


\subsection{Características da paisagem}

A paisagem da microbacia foi analisada com base nas características geométricas, topográficas e hidrográficas. Para a análise foram utilizadas combinações de metodologias quantitativas e qualitativas, visto que, segundo Pereira et al. (2018), a primeira permite a aquisição de dados numéricos utilizando mensurações de grandezas, enquanto a segunda possibilita a interpretação e a elaboração de opiniões sobre estes dados numéricos. A metodologia consistiu em 4 etapas (Figura 2), descritas com maior nível de detalhes a seguir:

Figura 2. Etapas para a aquisição das características da paisagem.

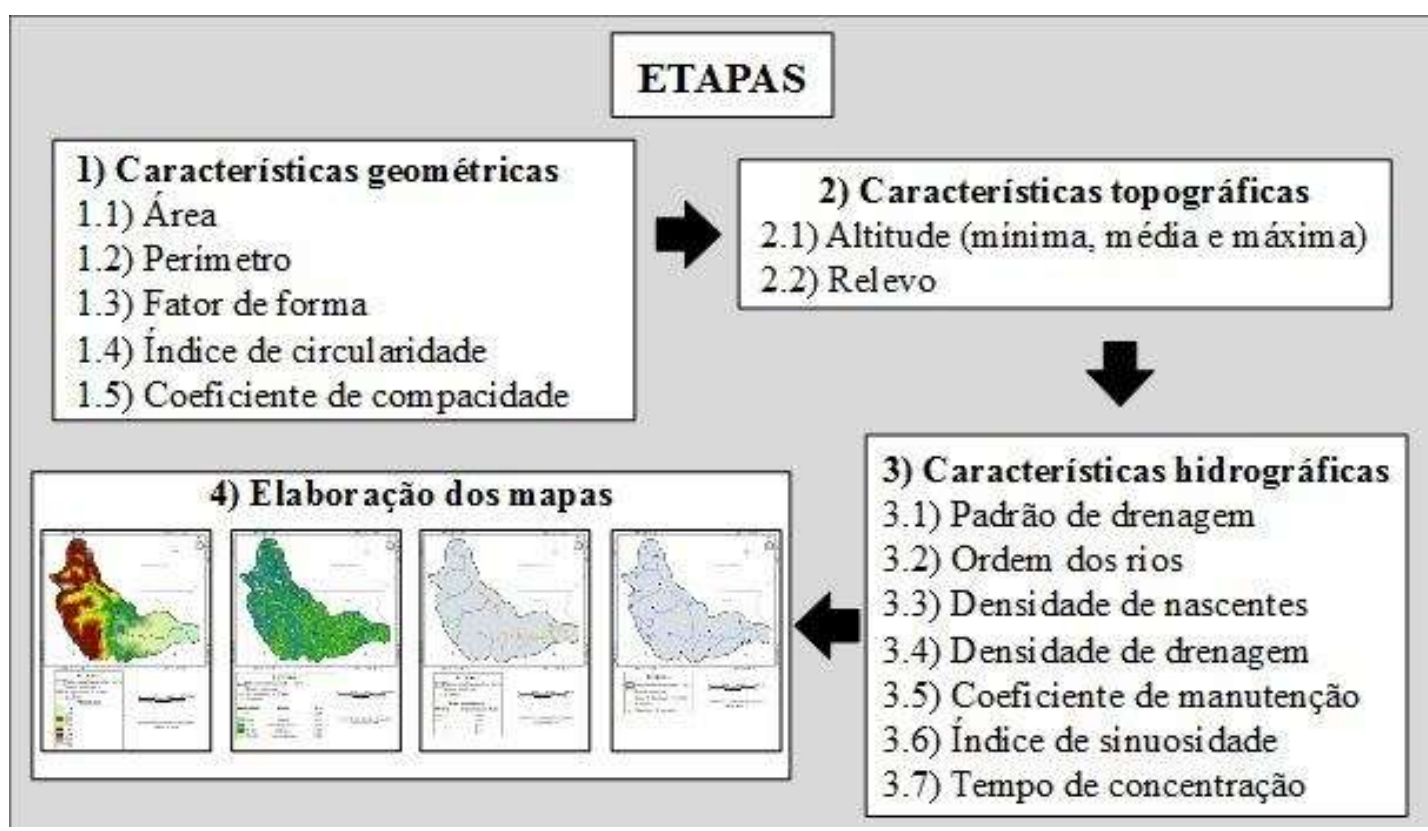

Fonte: Autores.

\section{1 a Etapa - Características geométricas}

Área e perímetro: a princípio delimitou-se a microbacia automáticamente com base na imagem altimétrica do satélite ALOS (ASF, 2017), utilizando o software QGIS e a ferramenta TauDEM (Pit Remove $\Rightarrow$ D8 Flow Directions $\Rightarrow$ D8 Contributing Area - 1모 versão $\Rightarrow$ Stream Definition By Threshold $\Rightarrow$ Edição do ponto de exutório $\Rightarrow$ D8 Contributing Area - $2^{\mathrm{a}}$ versão). Em seguida, o arquivo da delimitação da microbacia, gerado no formato matricial, foi convertido para o formato vetorial, dissolvido e suavizado, no software QGIS, e ajustado no software Google Earth, considerando as características hidrográficas e topográficas da paisagem. Por último, foram calculados a área e o perímetro com a ferramenta "calculadora de campo".

Fator de forma: é a relação entre a largura média e o comprimento axial da microbacia, e foi calculado com a equação 1 (Villela \& Mattos, 1975) e classificado de acordo com a literatura (Tabela 1).

$$
F=\frac{A}{L^{2}}
$$

Onde: $\mathrm{F}$ = fator de forma; $\mathrm{A}=$ área da microbacia $\left(\mathrm{km}^{2}\right) ; \mathrm{L}=$ comprimento do eixo da microbacia $(\mathrm{km})$.

Índice de circularidade: relaciona a área da microbacia com a área de um círculo de perímetro igual ao da área da microbacia, e foi calculado com a equação 2 (Christofoletti, 1980) e classificado de acordo com a literatura (Tabela 1).

$$
I c=\frac{12,57 x A}{P^{2}}
$$


Onde: $\mathrm{Ic}=$ índice de circularidade; $\mathrm{A}=$ área da microbacia $\left(\mathrm{km}^{2}\right) ; \mathrm{P}=$ perímetro da microbacia $(\mathrm{km})$.

Coeficiente de compacidade: é a relação entre o perímetro da microbacia e a circunferência de um círculo com área igual a microbacia, e foi calculado com a equação 3 (Villela \& Mattos, 1975) e classificado de acordo com a literatura (Tabela 1).

$$
\begin{array}{ll}
K c & =0,28 x \frac{P}{\sqrt{A}} \quad \text { (Equação 3) }
\end{array}
$$

Onde: $\mathrm{Kc}=$ coeficiente de compacidade; $\mathrm{A}=$ área da microbacia $\left(\mathrm{km}^{2}\right) ; \mathrm{P}=$ perímetro da microbacia $(\mathrm{km})$.

Tabela 1. Classificação dos parâmetros geométricos.

\begin{tabular}{ccc}
\hline Parâmetro & Limite & Classe \\
\hline \multirow{2}{*}{ Fator de forma $^{1}$} & $<0,50$ & Não sujeito a inundações \\
& $0,50-0,75$ & Tendência média a inundações \\
& $0,76-1,00$ & Sujeito a inundações \\
\hline \multirow{2}{*}{ Índice de circularidade $^{2}$} & $<0,51$ & Forma alongada \\
& $0,51-0,75$ & Forma intermediária \\
& $0,76-1,00$ & Forma circular \\
\hline \multirow{2}{*}{ Coeficiente de compacidade ${ }^{1}$} & $1,00-1,25$ & Alta propensão a inundações \\
& $1,26-1,50$ & Tendência média a inundacõos \\
\hline
\end{tabular}

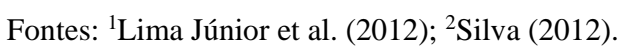

\section{$2^{\text {a }}$ Etapa - Características topográficas}

Altitude: é a elevação vertical em relação a um ponto de referência, o nível do mar (nível zero). As altitudes mínima e máxima foram obtidas diretamente da imagem altimétrica, e a altitude média foi estimada com o auxílio da ferramenta "estatística por zona", do software QGIS.

Relevo: representa a forma da superfície do planeta, e pode ser classificado de acordo com a declividade em plano (0-3\%), suave ondulado (3-8\%), ondulado (8-20\%), forte ondulado (20-45\%), montanhoso (45-75\%) e escarpado (> 75\%) (Santos, et al., 2013). A declividade da paisagem na microbacia foi calculada com a ferramenta "modelo digital de elevação", e também foi utilizada para obter informações sobre a influência na propagação de incêndios e aptidão à mecanização agrícola (Tabela 2).

\begin{tabular}{|c|c|c|}
\hline Parâmetro & Classe & Declividade (\%) \\
\hline \multirow{5}{*}{ Influência a propagação de incêndios ${ }^{1}$} & Baixa & $\leq 15$ \\
\hline & Moderada & $16-25$ \\
\hline & Alta & $26-35$ \\
\hline & Muito alta & $36-45$ \\
\hline & Extremamente alta & $>45$ \\
\hline \multirow{5}{*}{ Aptidão à mecanização agrícola ${ }^{2}$} & Extremamente apta & $0-5,0$ \\
\hline & Muito apta & $5,1-10,0$ \\
\hline & Apta & $10,1-15,0$ \\
\hline & Moderadamente apta & $15,1-20,0$ \\
\hline & Não apta & $>20,0$ \\
\hline
\end{tabular}

Tabela 2. Classificação da influência da declividade na propagação de incêndios e aptidão à mecanização agrícola.

Fontes: ${ }^{1}$ Ribeiro, et al. (2008); ${ }^{2}$ Höfig \& Araújo-Júnior (2015).

\section{$3^{a}$ Etapa - Características hidrográficas}

Padrão de drenagem: refere-se a distribuição espacial da rede de drenagem na paisagem, e foi identificada por comparação 
com imagens disponibilizadas no trabalho de Parvis (1950). A rede de drenagem da microbacia foi gerada nos softwares Google Earth, TrackMaker Free e QGIS, no primeiro software foram gerados os rios com a ferramenta "adicionar caminho" e salvos os arquivos no formato KML, no segundo software os rios foram unidos com a ferramenta "lápis", formando um único arquivo no formato KML, e no terceiro software o formato KML foi convertido para o formato SHP.

Ordem de drenagem: fornece informações sobre o grau de ramificação da rede de drenagem e a complexidade do ecossistema aquático. Este parâmetro foi calculado com a ferramenta "Strahler", onde rios de $1^{\mathrm{a}}$ ordem são formados diretamente das nascentes, os rios de $2^{\mathrm{a}}$ ordem são formados quando dois rios de $1^{\mathrm{a}}$ ordem se encontram, os rios de $3^{\mathrm{a}}$ ordem são formados quando dois rios de $2^{\mathrm{a}}$ ordem se encontram, assim, sucessivamente (Strahler, 1954). Em seguida, foi classificado de acordo com a literatura (Tabela 3).

Densidade de nascentes: corresponde a quantidade de nascente por unidade de área, indicando o potencial hídrico da região, quanto maior a densidade, mais elevado o potencial (Cherem, et al., 2020). Este parâmetro foi calculado com a equação 4 (Christofoletti, 1980) e classificado de acordo com dados da literatura (Tabela 3).

$$
\begin{aligned}
& D n=\frac{N}{A} \\
& \text { (Equação 4) }
\end{aligned}
$$

Onde: $\mathrm{Dn}=$ densidade de nascentes $\left(\right.$ nascentes $\left.\mathrm{km}^{-2}\right) ; \mathrm{N}=$ número de nascentes; $\mathrm{A}=$ área da microbacia $\left(\mathrm{km}^{2}\right)$.

Densidade de drenagem: denota o grau de desenvolvimento do sistema de drenagem, e fornece uma indicação da eficiência de drenagem da microbacia (Villela \& Mattos, 1975). Este parâmetro foi calculado com a equação 5 (Horton 1932) e classificado de acordo com dados da literatura (Tabela 3).

$$
D d=\frac{L}{A}
$$

Onde: $\mathrm{Dd}=$ densidade de drenagem $\left(\mathrm{km} \mathrm{km}^{-2}\right) ; \mathrm{L}=$ comprimento da rede de drenagem $(\mathrm{km}) ; \mathrm{A}=$ área da microbacia $\left(\mathrm{km}^{2}\right)$.

Coeficiente de manutenção: representa a área necessária que a microbacia deve ter para manter perene cada metro de canal de drenagem, e foi calculado com a equação 6 (Santos, et al., 2012).

$$
C m=\frac{1}{D d} x 1000
$$

Onde: $\mathrm{Cm}=$ coeficiente e manutenção $\left(\mathrm{m}^{2} \mathrm{~m}^{-1}\right)$; $\mathrm{Dd}=$ densidade de drenagem $\left(\mathrm{km} \mathrm{km}^{-2}\right)$.

Índice de sinuosidade: é um fator controlador da velocidade do fluxo hídrico, quanto maior a sinuosidade, menor a velocidade, e foi calculado com a equação 7 (Villela \& Mattos, 1975). Este parâmetro foi classificado de acordo com a literatura (Tabela 3).

$$
\begin{aligned}
& I s=\frac{L-D v}{L} \times 100 \\
& \text { (Equação 7) }
\end{aligned}
$$

Onde: Is = índice de sinuosidade (\%); $\mathrm{L}=$ comprimento do canal principal $(\mathrm{km}) ; \mathrm{Dv}=$ distância vetorial do canal principal $(\mathrm{km})$.

Tempo de concentração: é o tempo necessário para que a água se desloque do ponto mais distante até o ponto de controle (exutório), e foi calculada com a equação 8 (Kirpich, 1940, citado por Targa, et al., 2012).

$$
T C=57 x\left(\frac{L^{3}}{H}\right)^{0,385}
$$

Onde: $\mathrm{Tc}=$ tempo de concentração (minutos); $\mathrm{L}=$ comprimento do talvegue principal $(\mathrm{km}) ; \mathrm{H}=$ desnível entre a parte mais elevada e a seção de controle (m). 
Tabela 3. Classificação das características hidrográficas.

\begin{tabular}{|c|c|c|c|}
\hline Parâmetro & Unidade & Classe & Limite \\
\hline \multirow{4}{*}{ Ordem dos rios ${ }^{1}$} & \multirow{4}{*}{ Unidades } & Improvável habitat de peixes & 1 \\
\hline & & Baixas condições para habitação Moderadas & 2 \\
\hline & & condições para habitação Elevadas condições & 3 \\
\hline & & para habitação & $\geq 4$ \\
\hline \multirow{4}{*}{ Densidade de nascentes $^{2}$} & \multirow{4}{*}{ Nascentes $\mathrm{km}^{-2}$} & Baixa & $<3$ \\
\hline & & Média & $3-7$ \\
\hline & & Alta & $7-15$ \\
\hline & & Muito alta & $>15$ \\
\hline \multirow{4}{*}{ Densidade de drenagem $^{3}$} & \multirow{4}{*}{$\mathrm{km} \mathrm{km}^{-2}$} & Baixa & $<0,50$ \\
\hline & & Média & $0,50-2,00$ \\
\hline & & Alta & $2,00-3,50$ \\
\hline & & Muito alta & $>3,50$ \\
\hline \multirow{5}{*}{ Índice de sinuosidade ${ }^{4}$} & \multirow{5}{*}{$\%$} & Muito reto & $<20$ \\
\hline & & Reto & $20-29$ \\
\hline & & Divagante & $30-39$ \\
\hline & & Sinuoso & $40-50$ \\
\hline & & Muito sinuoso & $>50$ \\
\hline
\end{tabular}

Fontes: ${ }^{1}$ Adaptado de Fairfull \& Witheridge (2003); ${ }^{2}$ Lollo (1995); ${ }^{3}$ Beltrame (1994); ${ }^{4}$ Romero, Formiga \& Marcuzzo (2017).

\section{$4^{\text {a }}$ Etapa - Elaboração dos mapas}

Os mapas foram elaborados no software QGIS, utilizado a ferramenta "novo compositor de impressão", e como referência, o Sistema de Coordenadas Geográficas e o Datum WGS 84.

\section{Resultados e Discussão}

\subsection{Características geométricas}

A microbacia tem área de $8,64 \mathrm{~km}^{2}$, perímetro de $15,26 \mathrm{~km}$, fator de forma de 0,22 , índice de circularidade de 0,47 e coeficiente de compacidade correspondente a 1,45. Esses resultados denotam que a microbacia tem formato alongado e baixa a média suscetibilidade a inundações, em condições de cobertura com vegetação nativa e precipitações normais.

O formato da microbacia está associado com a declividade do terreno, segundo Schumm (1956), quanto mais íngreme a encosta em que as microbacias se desenvolvem, mais espaçados estão os canais de drenagem, resultando em formas de microbacias mais alongadas. Uma microbacia com formato alongado tem menor suscetibilidade a enchentes em relação a uma microbacia de formato circular, com área igual, por duas razões, a contribuição dos tributários atinge o curso d'água principal em vários pontos ao longo do mesmo, e existe menor probabilidade de que precipitações ocorram em toda a sua extensão simultaneamente (Villela \& Mattos, 1975).

\subsection{Características topográficas}

Os valores de altitude variam de 228 a 322 m, com média de 265 m (Figura 3), e amplitude altimétrica de 94 m. Diversos fatores como a transpiração, precipitação e evaporação, estão relacionados com a variação altimétrica, a qual pode influenciar tanto na temperatura média, quanto no regime de chuvas de uma bacia hidrográfica (Teodoro, et al., 2007). Logo, é possível utilizar informações de altitudes para auxiliar na seleção de espécies de interesse econômico, para fortalecer a agricultura local (Silva Neto, et al., 2013). 
Figura 3. Altitude da microbacia do rio Mutum, Amazônia Ocidental, Brasil.

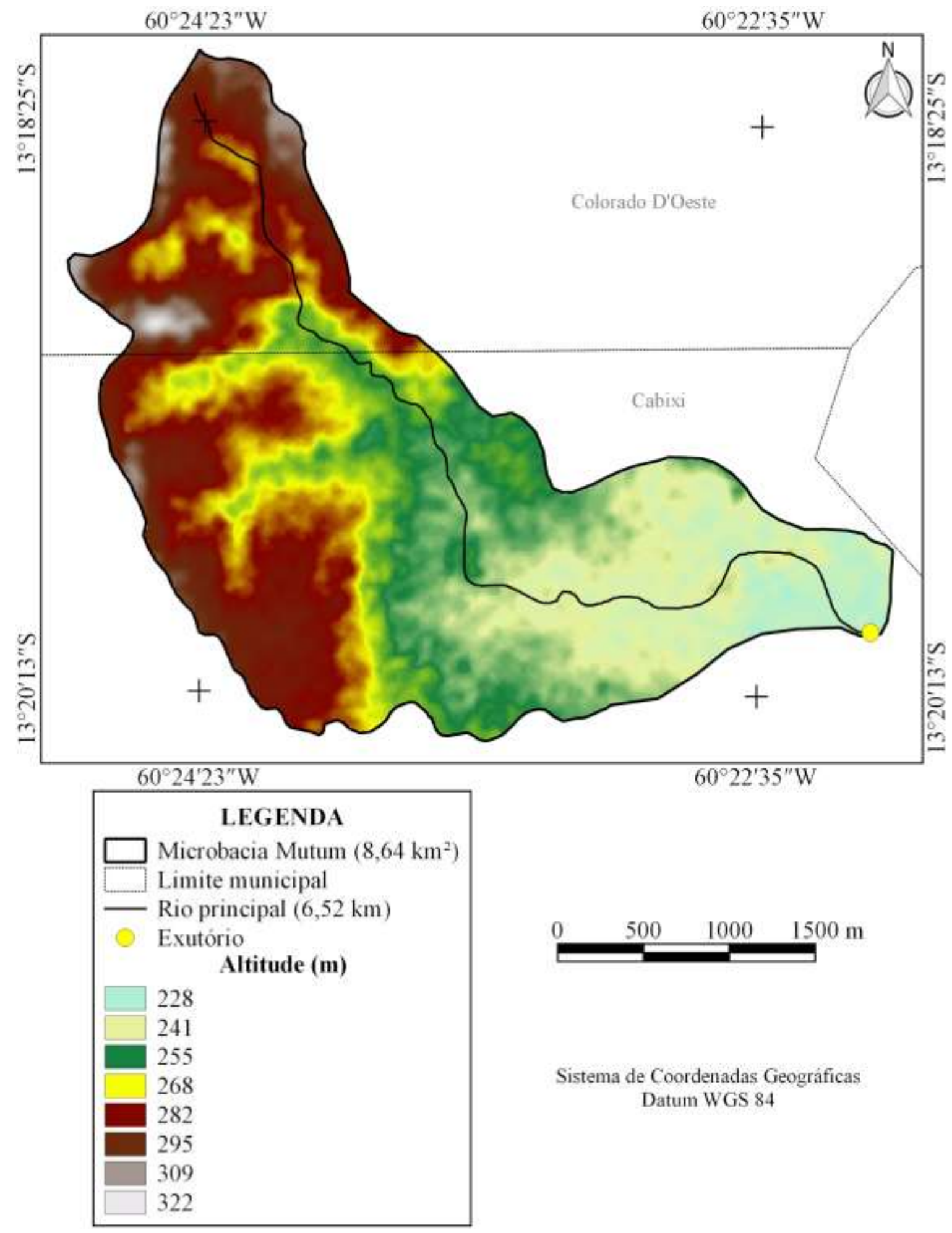

Fonte: Autores.

Existem diversas espécies vegetais de interesse econômico que podem ser encontradas na mesma faixa de altitude da microbacia em estudo, como pode ser observado no trabalho de Bourke (2010): Abelmoschus esculentus (quiabo), Anacardium occidentale (cajú), Ananas comosus (abacaxi), Annona muricata (graviola), Annona reticulata (Ata), Annona squamosa (pinha), Arachis hypogaea (amendoim), Artocarpus altilis (Fruta-pão), Artocarpus heterophyllus (jaca), Averrhoa carambola (carambola), Basella alba (bertalha), Bixa orellana (urucum), Brassica oleracea cv. Group Broccoli (brócolis), Brassica oleracea 
cv. Group Cauliflower (couve-flor), Carica papaya (mamão), Capsicum annuum cv. group Grossum (pimentão), Capsicum frutescens (pimenta malagueta), Ceiba pentandra (sumaúma), Citrus aurantifolia (lima), Citrullus lanatus (melancia), Citrus limon (limão), Citrus paradisi (toranja), Citrus reticulata (tangerina), Citrus sinensis (laranja), Cucumis melo (melão), Cucumis sativus (pepino), Coffea canephora var. robusta (café conilon), Cucurbita moschata (abóbora-cheirosa), Cocos nucifera (coco), Cymbopogon citratus (capim cidreira), Dioscorea alata (cará) Dioscorea bulbifera (cará-do-ar), Dioscorea esculenta (inhame), Eugenia uniflora (pitanga), Glycine max (soja), Helianthus annuus (girassol), Hevea brasiliensis (seringueira), Ipomoea batatas (batata doce), Lycopersicon esculentum (tomate), Mangifera indica (manga), Manihot esculenta (mandioca), Mentha spicata (hortelã), Musa sp. (banana), Oryza sativa (arroz), Passiflora edulis f. flavicarpa (maracujá-amarelo), Persea americana (abacate), Petroselinum crispum (salsa), Piper nigrum (pimenta-do-reino), Phaseolus vulgaris (feijão), Psidium cattleianum (araçáamarelo), Psidium guajava (goiaba), Ricinus communis (mamona), Rorippa nasturtium-aquaticum (agrião), Saccharum officinarum (cana-de-açúcar), Sechium edule (chuchu), Spondias cytherea (cajá-manga), Theobroma cacao (cacau), Xanthosoma sagittifolium (taioba), Zea mays (milho) e Zingiber officinale (gengibre). A grande diversidade de espécies de interesse econômico denota que as propriedades rurais podem ter várias fontes de renda, e assim, reduzir o risco financeiro.

A região tem relevos planos $(16,44 \%)$ a forte ondulados $(2,08 \%)$, com predominância de suave ondulado $(50,81 \%)$ e ondulado (30,67\%) (Figura 4). Em relevo plano o escoamento superficial é lento a muito lento, e vai aumentando com a declividade, chegando a ser considerado rápido no relevo forte ondulado (Lepsch, et al., 2015). Logo, o aumento da declividade também potencializa as perdas de solo, água, matéria orgânica e nutrientes (Bertoni \& Lombardi Neto, 2014; Lobato, et al., 2019), havendo a necessidade de se adotar práticas de manejo conservacionista do solo nos sistemas agropecuários, para mitigar esse problema, principalmente nos relevos ondulado e forte ondulado.

As práticas de manejo conservacionista do solo são classificadas em caráter vegetativo, edáfico e mecânico, e tem objetivos relacionados a proteção contra o impacto direto das gotas de chuva na superfície do solo, aumento ou manutenção da fertilidade do solo, redução do escoamento superficial e aumento da infiltração de água no solo (Bertoni \& Lombardi Neto, 2014). Estas práticas podem ser utilizadas de forma integrada, para mitigar os problemas ocasionados pela erosão hídrica e permitir o aumento da produtividade agrícola, além de ser uma importante estratégia para reduzir os riscos ocasionados por estiagens (Pittelkow, et al., 2014).

A declividade na microbacia varia de 0 a $40 \%$ (Figura 4), indicando que a região tem 92,93\% de sua área classificada como de baixa propensão na influencia a propagação de incêndios, $6,48 \%$ como moderada, e apenas $0,59 \%$ como alta a muito alta. A predominância da classe baixa é desejável visto que na microbacia existem aproximadamente 20 estabelecimentos agropecuários privados, que poderiam ter prejuízos financeiros em função de incêndios descontrolados. Apesar da baixa propensão ocorrer na maior parte da área da microbacia, é importante lembrar que existem regiões mais íngremes, que exercem alta influência a propagação de incêndios, portanto, é recomendado a adoção de ações preventivas e inibitórias combinadas (educação ambiental, fiscalização) para reduzir os riscos de incêndios, como descrito por Medeiros \& Fiedler (2004). 
Figura 4. Relevo da microbacia do rio Mutum, Amazônia Ocidental, Brasil.

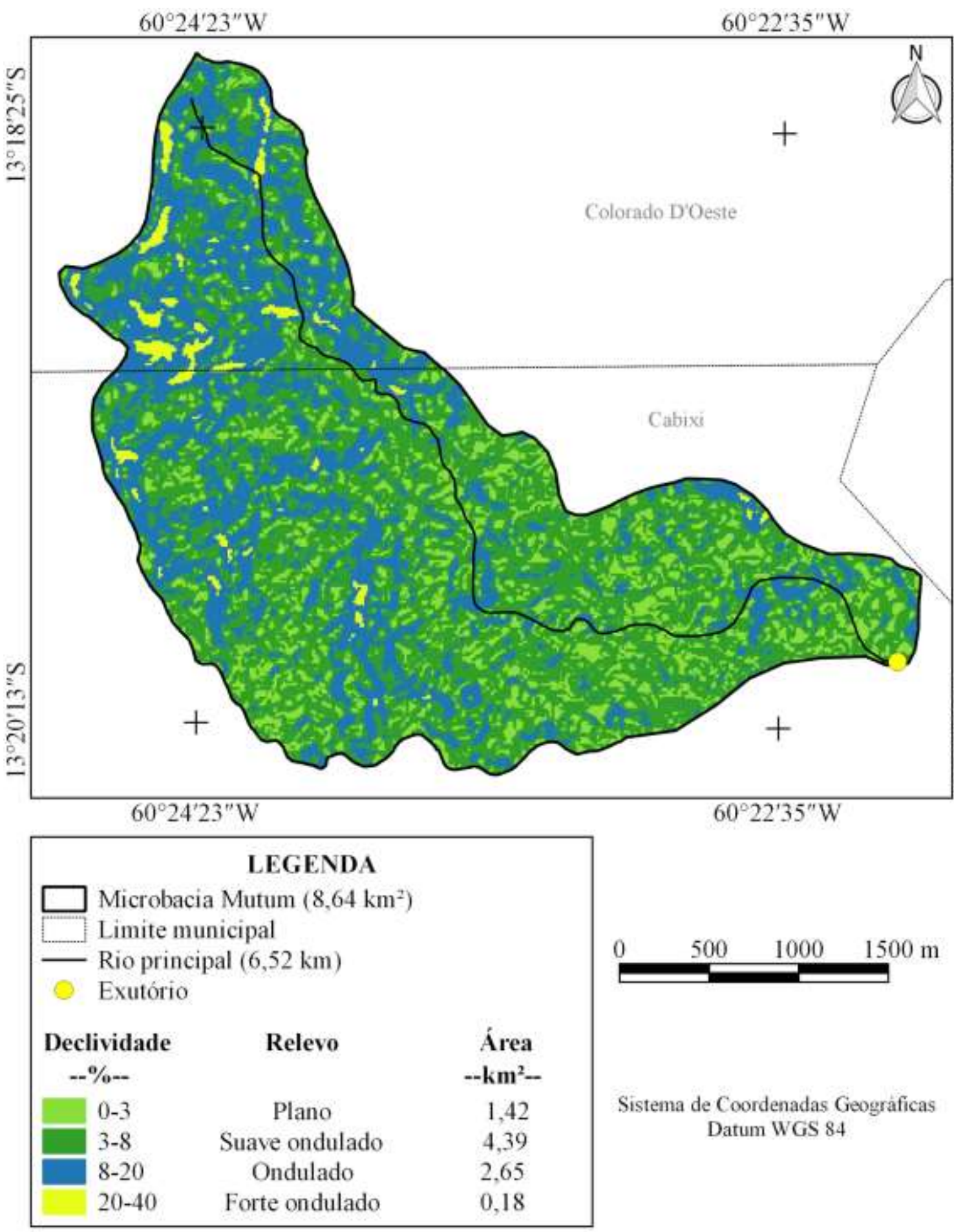

Fonte: Autores.

Aproximadamente $92,94 \%$ da área da microbacia é considerada apta a extremamente apta à mecanização agrícola. Essa característica associada a presença de Argissolos Vermelhos eutróficos em 98,32\% da área da microbacia, permite o avanço de sistemas de plantios mecanizados e explica porque as áreas plantadas com soja e milho vem crescendo na região nas últimas décadas (Tabela 4). 
Tabela 4. Área cultivada com soja e milho nos municípios de Cabixi/RO e Colorado D`Oeste/RO, no período de 1990 a 2019.

\begin{tabular}{|c|c|c|c|c|c|}
\hline \multirow{2}{*}{ Município } & \multirow{2}{*}{ Cultura } & \multicolumn{4}{|c|}{ Ano } \\
\hline & & 1990 & 2000 & 2010 & 2019 \\
\hline \multirow{2}{*}{ Cabixi/RO } & Soja & 0 & 200 & 10.920 & 24.630 \\
\hline & Milho & 3.500 & 5.500 & 5.500 & 20.020 \\
\hline \multirow[t]{2}{*}{ Colorado D'Oeste/RO } & Soja & 0 & 400 & 3.000 & 3.250 \\
\hline & Milho & 10.400 & 6.000 & 2.800 & 1.550 \\
\hline
\end{tabular}

Fonte: IBGE $\left(2021^{\mathrm{a}}\right)$.

\subsection{Características hidrográficas}

A microbacia do rio Mutum tem rede de drenagem de 17,30 km, padrão dendrítico, $4^{\mathrm{a}}$ ordem de drenagem (Figura 5), 3,24 nascentes $\mathrm{km}^{-2}$ (Figura 6), densidade de drenagem de 2,00 km km${ }^{-2}$, coeficiente de manutenção de 499,4 $\mathrm{m}^{2} \mathrm{~m}^{-1}$, índice de sinuosidade de $22,70 \%$ e tempo de concentração de $1,78 \mathrm{~h}$.

Os padrões dendríticos são muito comuns no estado de Rondônia, como pode ser visto nas microbacias dos rios Enganado (Moreto, et al., 2019), Alto Rio Escondido (Vendruscolo, et al., 2020a), Médio Rio Escondido (Vendruscolo, et al., 2020b), São Jorge (Pacheco, et al., 2020), Jacuri (Panza, et al., 2020), Gavião (Donegá, et al., 2021) e Tamarupá (Vendruscolo, et al., 2021). Esse padrão se desenvolve em regiões onde a rocha localizada abaixo do rio não tem tecido ou estrutura particular (exemplos: granito, gnaisse, rocha vulcânica e rocha sedimentar que não foi dobrada), e pode ser erodida com a mesma facilidade em todas as direções (Earle \& Panchuk, 2019).

As densidades de nascentes e de drenagem são consideradas médias (Tabela 3), indicando que a região tem uma rede de drenagem em pleno desenvolvimento, com boa capacidade de gerar novos cursos d'água e considerável disponibilidade hídrica. É importante ressaltar que o aumento da densidade de drenagem eleva a capacidade da microbacia fazer escoamentos rápidos no exutório, resultando em deflúvios mais baixos nos períodos de estiagem (Finkler, 2010). Em face ao exposto, constata-se a necessidade de adotar planos de manejo que favoreçam a infiltração de água no solo, para o abastecimento do lençol freático, e assim, manter certa regularidade na vazão dos rios ao longo do ano.

As práticas de manejo conservacionistas citadas anteriormente, para mitigar os problemas relacionados à erosão do solo, são eficientes para favorecer a infiltração de água no solo. Contudo, segundo Tambosi et al. (2015), também é necessário considerar a manutenção da floresta nativa em quantidade adequada em diferentes posições do relevo, nos topos dos morros para o abastecimento do lençol freático, nas encostas para contenção de processos erosivos, nas zonas ripárias para contenção de sedimentos e contaminantes, e nos intervales para auxiliar nas funções descritas, e assim, garantir água em quantidade e qualidade. Logo, recomendam-se estudos referentes à análise da cobertura do solo na microbacia do rio Mutum, para identificar e delimitar áreas prioritárias para a conservação e/ou recuperação da vegetação nativa. 
Figura 5. Rede de drenagem e ordem dos rios na microbacia do rio Mutum, Amazônia Ocidental, Brasil.

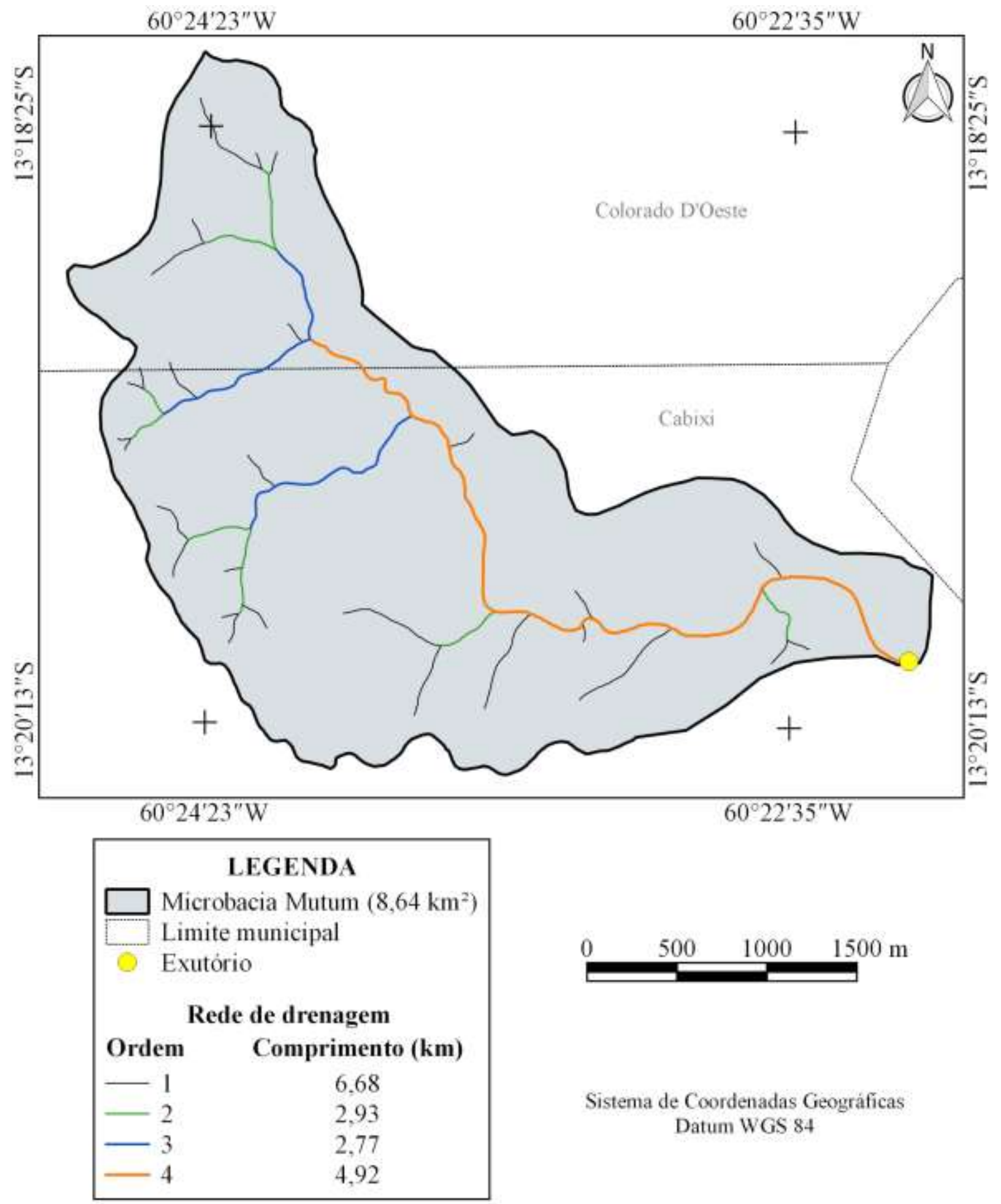

Fonte: Autores. 
Figura 6. Distribuição espacial das nascentes na microbacia do rio Mutum, Amazônia Ocidental, Brasil.

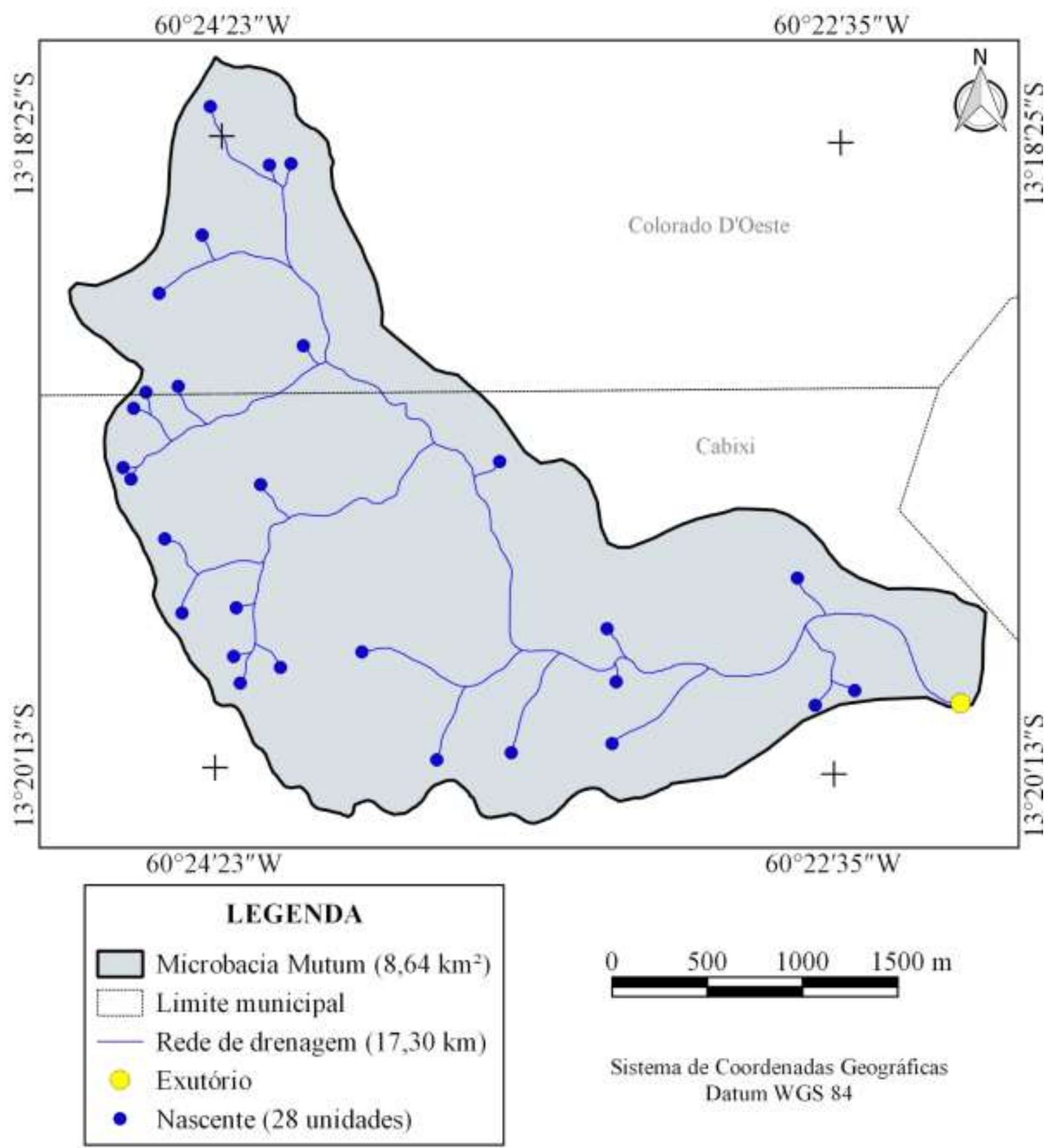

Fonte: Autores.

O número de ordens de drenagem sugere elevadas condições para habitações de peixes (Tabela 3). A prática da piscicultura tem-se desenvolvido nos municípios de Cabixi e Colorado D’Oeste, durante o período de 2013 a 2019 , destacando-se as espécies conhecidas como tambaqui e jatuarana (Tabela 5). Logo, o número de ordens e a prática da piscicultura confirma o potencial para o desenvolvimento desta atividade na região. 
Tabela 5. Produção de peixes em piscicultura nos municípios de Cabixi e Colorado D’Oeste, estado de Rondônia, no período de 2013 a 2019.

\begin{tabular}{|c|c|c|c|c|c|c|c|c|}
\hline \multirow{2}{*}{ Município } & \multirow{2}{*}{ Espécie } & \multicolumn{7}{|c|}{ Ano } \\
\hline & & 2013 & 2014 & 2015 & 2016 & 2017 & 2018 & 2019 \\
\hline & & ---- & - & P & rodução & t)-----.- & ----- & ------ \\
\hline \multirow{9}{*}{ Cabixi } & Jatuarana & 0,00 & 0,00 & 11,33 & 17,24 & 14,06 & 11,54 & 11,26 \\
\hline & Pacu e patinga & 3,00 & 3,00 & 5,62 & 7,82 & 6,57 & 5,96 & 5,49 \\
\hline & Piau e piauçu & 2,00 & 2,00 & 0,00 & 0,00 & 0,00 & 0,00 & 0,00 \\
\hline & Pintado* & 3,50 & 3,50 & 9,36 & 13,09 & 11,20 & 9,00 & 9,89 \\
\hline & & & & & & & & \\
\hline & Pirarucu & 2,50 & 12,96 & 16,85 & 0,19 & 0,20 & 0,29 & 0,30 \\
\hline & Tambaqui & 42,00 & 73,44 & 14,14 & 19,54 & 16,11 & 14,04 & 13,75 \\
\hline & Tilápia & 2,00 & 2,00 & 0,00 & 0,00 & 0,00 & 0,00 & 0,00 \\
\hline & Total & 55,00 & 96,90 & 57,30 & 57,88 & 48,14 & 40,82 & 40,69 \\
\hline \multirow{11}{*}{$\begin{array}{l}\text { Colorado } \\
\text { D’Oeste }\end{array}$} & Curimatã & 3,00 & 0,00 & 0,00 & 0,00 & 0,00 & 0,00 & 0,00 \\
\hline & Jatuarana & 0,00 & 0,00 & 34,61 & 20,25 & 16,05 & 13,70 & 13,27 \\
\hline & Lambari & 0,80 & 0,00 & 0,00 & 0,00 & 0,00 & 0,00 & 0,00 \\
\hline & Pacu e patinga & 0,00 & 0,00 & 17,31 & 11,51 & 8,84 & 6,81 & 6,61 \\
\hline & Piau e piauçu & 2,00 & 0,00 & 0,00 & 0,00 & 0,00 & 0,00 & 0,00 \\
\hline & Pintado* & 18,00 & 18,00 & 28,85 & 15,17 & 12,51 & 9,59 & 10,44 \\
\hline & Pirarucu & 4,00 & 93,42 & 51,92 & 25,46 & 5,94 & 2,53 & 2,76 \\
\hline & Tambacu e tambatinga & 70,00 & 0,00 & 0,00 & 0,00 & 0,00 & 0,00 & 0,00 \\
\hline & Tambaqui & 493,00 & 529,36 & 444,21 & 266,52 & 200,22 & 161,83 & 164,64 \\
\hline & Tilápia & 6,00 & 0,00 & 0,00 & 0,00 & 0,00 & 0,00 & 0,00 \\
\hline & Tucunaré & 12,00 & 0,00 & 0,00 & 0,00 & 0,00 & 0,00 & 0,00 \\
\hline
\end{tabular}




\section{Total}

$$
608,80
$$

640,7

576,89

338,92

243,56

194,46

197,72

*Cachara, cachapira, pintachara e surubim. Fonte: IBGE, 2021 b.

O coeficiente de manutenção denota que são necessários 499,4 $\mathrm{m}^{2}$ de área na microbacia para manter $1 \mathrm{~m}$ de curso d'água. Esse valor encontra-se mais elevado do que os observados nas microbacias Alto Rio Escondido $\left(234,1 \mathrm{~m}^{2} \mathrm{~m}^{-1}\right)\left(\mathrm{Ven}^{-}\right.$ druscolo, et al., 2020a) e Médio Rio Escondido (246,0 $\mathrm{m}^{2} \mathrm{~m}^{-1}$ ) (Vendruscolo, et al., 2020b), e mais baixos que os constatados nas microbacias Gavião (1.250,0 $\left.\mathrm{m}^{2} \mathrm{~m}^{-1}\right)$ (Donegá, et al., 2021) e Santa Teresinha (2.439,0 $\left.\mathrm{m}^{2} \mathrm{~m}^{-1}\right)$ (Soares, et al., 2019b). Ao ordenar as microbacias citadas em ordem decrescente, com relação a declividade média, tem-se Alto Rio Escondido, Médio Rio Escondido, Mutum, Gavião e Santa Teresinha, demonstrando que o valor do coeficiente de manutenção diminui à medida que a declividade é reduzida. O coeficiente de manutenção é calculado com base na densidade de drenagem, que tende a se elevar com a inclinação do terreno (Vendruscolo, et al., 2020a; Vendruscolo, et al., 2020b).

O índice de sinuosidade confirma a presença de um canal principal reto na microbacia do rio Mutum. Esta característica é igual à observada no canal principal da microbacia Santa Teresinha (Soares, et al., 2019b), e semelhante às constatadas nos canais principais das microbacias Jacuri (Panza, et al., 2020) e Gavião (Donegá, et al., 2021). Quanto mais reto for o canal, mais rápido é a velocidade de escoamento (Villela \& Mattos, 1975), sendo estimado uma velocidade média de 3,66 km $\mathrm{h}^{-1}$ para a microbacia em estudo.

O tempo de concentração de 1,78 h é baixo se levar em consideração que as precipitações na região ultrapassam facilmente esse tempo. Em trabalho realizado por Fietz et al. (2011), são constatadas precipitações que duram até 24 h no município de Colorado D’Oeste. Portanto, podem ocorrer inundações quando houver a combinação de precipitações intensas que ultrapassam o tempo de $1,78 \mathrm{~h}$, visto que toda a área da microbacia contribui simultaneamente com escoamento na seção de deságue (exutório).

\section{Conclusão}

A microbacia do rio Mutum tem área de $8,64 \mathrm{~km}^{2}$, perímetro de $15,26 \mathrm{~km}$, formato alongado, altitudes de 228 a 322 $\mathrm{m}$, predominância de relevos suave ondulado e ondulado, baixa influencia a propagação de incêndios e aptidão à mecanização em 92,94\% da área, médias densidade de nascentes e drenagem, coeficiente de manutenção de $499,4 \mathrm{~m}^{2} \mathrm{~m}^{-1}$, canal principal reto e baixo tempo de concentração.

Na região existe grande potencial para o desenvolvimento de atividades agropecuárias, contudo, é necessário adotar práticas de manejo conservacionista do solo para mitigar possíveis problemas ocasionados por processos de erosão, e favorecer o abastecimento do lençol freático por meio da infiltração de água da chuva.

É recomendado um estudo sobre a dinâmica temporal e espacial da cobertura do solo na microbacia do rio Mutum, para analisar a qualidade e a quantidade de vegetação nativa em áreas de Reservas Legais e Áreas de Preservação Permanente, e assim, delimitar as áreas prioritárias para a manutenção e/ou recuperação dessa vegetação.

As técnicas de sensoriamento remoto permitiram a aquisição de informações geométricas, topográficas e hidrográficas da microbacia, e podem ser utilizadas para auxiliar o planejamento e o gerenciamento dos recursos naturais nos estabelecimentos agropecuários.

\section{Referências}

Alvares, C. A, Stape, J. L., Sentelhas, P. C., Gonçalves, L. M., \& Sparovek, G. (2014). Köppen's climate classification map for Brazil. Meteorologische Zeitschrift, 22(6), 711-728. 10.1127/0941-2948/2013/0507

ASF - Alaska Satellite Facility (2017). Imagem altimétrica. https://www.asf.alaska.edu/ 
Beltrame, A.V. (1994). Diagnóstico do meio ambiente físico de bacias hidrográficas: modelo de aplicação. Florianópolis: Universidade Federal de Santa Catarina.

Bertoni, J., \& Lombardi Neto, F. (2014). Conservação do solo. Editora Ícone.

Bourke, R. M. (2010). Altitudinal limits of 230 economic crop species in Papua New Guinea. In: Haberle, S.G., Stevenson, J., \& Prebble, M. (eds). Altered Ecologies: Fire, Climate and Human Influence on Terrestrial Landscapes. Canberra: The Australian National University.

Brasil. (1997). Lei no 9.433, de 8 de janeiro de 1997. Institui a Política Nacional de Recursos Hídricos, cria o Sistema Nacional de Gerenciamento de Recursos Hídricos, regulamenta o inciso XIX do art. 21 da Constituição Federal, e altera o art. $1^{\circ}$ da Lei n ${ }^{\circ}$ 8.001, de 13 de março de 1990, que modificou a Lei nº 7.990 , de 28 de dezembro de 1989. www.planalto.gov.br/ccivil_03/leis/L9433.htm

Cavalheiro, W. C. S., \& Vendruscolo, J. (2019). Importância de estudos em bacias hidrográficas para o manejo sustentável dos recursos hídricos em Rondônia. Revista Geográfica Venezolana, especial, 256-264. http://www.saber.ula.ve/handle/123456789/46164

Cherem, L. F. S., Faria, S. D., Zancopé, M. H. C., Sordi, M. V., Nunes, E. D., \& Rosa, L. E. (2020). Análise morfométrica em bacias hidrográficas. In. Magalhães Júnior, A.P., \& Barros, L.F.P. Hidrogeomorfologia: formas, processos e registros sedimentares fluviais. Rio de Janeiro-RJ: Bertrand Brasil.

Christofoletti, A. (1980). Geomorfologia. (2a ed.), Edgard Blucher.

Donegá, M. V. B., Souza, T. W. S., Lima, M. M., Panza, M. R., Pacheco, F. M. P., Saraiva, J. G., Cavalheiro, W. C. S., \& Vendruscolo, J. (2021). Caracterização hidrogeomorfométrica da microbacia do rio Gavião, Amazônia Ocidental, Brasil. Research, Society and Development, 10(1), 1-14. 10.33448/rsd-v10i1.11844

Earle, E., \& Panchuk, K. (2019). Physical Geology. (2a ed.), Victoria, B.C.: BCcampus. https://opentextbc.ca/physicalgeology2ed/

Fairfull, S., \& Witheridge, G. (2003). Why do Fish Need to Cross the Road? Fish Passage Requirements for Waterway Crossings. Sydney: NSW Fisheries.

Fietz, C. R., Comunello, É., Cremon, C., Dallacort, R., \& Pereira, S. B. (2011). Chuvas intensas no Estado de Mato Grosso. Dourados-MS: Embrapa Agropecuária Oeste. https://www.infoteca.cnptia.embrapa.br/bitstream/doc/915074/1/DOC2011104.pdf

Finkler, R. (2010). Planejamento, manejo e gestão de bacias. Brasília-DF: Agência Nacional de Águas.

Franca, R. R. (2015). Climatologia das chuvas em Rondônia - período 1981-2011. Revista Geografias, 11(1), 44-58. https://periodicos.ufmg.br/index.php/geografias/article/view/13392/10624

Höfig, P., \& Araujo-Junior, C. F. (2015). Classes de declividade do terreno e potencial para mecanização no estado do Paraná. Coffee Science, 10(2), 195-203. https://www.researchgate.net/publication/277597916_classes_de_declividade_do_terreno_e_potencial_para_mecanizacao_no_estado_do_parana

Horton, R. E. (1932). Drainage basin characteristics. Transactions, American Geophysical Union, 13(1), 350-361.

IBGE - Instituto Nacional de Geografia e Estatística (2021a). Produção agrícola municipal. https://sidra.ibge.gov.br/Tabela/1612

IBGE - Instituto Brasileiro de Geografia e Estatística (2021b). Pesquisa da pecuária municipal: produção da aquicultura, por tipo de produto. https://sidra.ibge.gov.br/tabela/3940.

INCRA - Instituto Nacional de Colonização e Reforma Agrária (2018). Acervo fundiário. http://acervofundiario.incra.gov.br/acervo/acv.php.

Lepsch, I. F., Espindola, C. R., Vischi Filho, O. J., Hernani, L. C., \& Siqueira, D. S. (2015). Manual para levantamento utilitário e classificação de terras no sistema de capacidade de uso. Viçosa-MG: Sociedade Brasileira de Ciência do Solo.

Lima Júnior, J. C., Vieira, W. L., Macêdo, K. G., Souza, S. A., \& Nascimento, F. A. L. (2012). Determinação das características morfométricas da sub-bacia do Riacho Madeira Cortada, Quixelô, CE. VII Congresso Norte-Nordeste de Pesquisa e Inovação. Palmas-TO: Instituto Federal de Educação, Ciência e Tecnologia. p. 1-7.

Lobato, F. A. O., Andrade, E. M., Meireles, A. C. M., Santos, J. C. N., \& Lopes, J. F. B (2009). Perdas de solo e nutrientes em área de Caatinga decorrente de diferentes alturas pluviométricas. Revista Agro@mbiente On-line, 3(2), 65-71. 10.18227/1982-8470ragro.v3i2.247

Lollo, J. A. (1995). O uso da técnica de avaliação do terreno no processo de elaboração do mapeamento geotécnico: sistematização e aplicação na quadrícula de Campinas. São Carlos: Universidade de São Paulo.

Medeiros, M. B., \& Fiedler, N. C. (2004). Incêndios florestais no Parque Nacional da Serra da Canastra: desafios da biodiversidade. Ciência Florestal, 14(2), 157-168. 10.5902/198050981815

Moreto, R. F., Mira, S. F., Soares, G. S., Santos Junior, N. R. F., Cavalheiro, W. C. S, Vendruscolo, J., \& Rosa, D. M. (2019). Características geométricas, topográficas e hidrográficas da microbacia do rio Enganado, região sul da Amazônia Ocidental. Revista Geográfica Venezolana, especial, 110-124. http://www.saber.ula.ve/handle/123456789/46159

Pacheco, F. M. P., Vendruscolo, J., Ramos, F. H., Rodrigues, A. A. M., Cavalheiro, W. C. S., Hara, F. A. S., Rocha, K. J., \& Silva, G. N. (2020). Caracterização hidrogeomorfométrica da microbacia do rio São Jorge, Rondônia, Brasil. Brazilian Journal of Development, 6(1), 4219-4236. 10.34117/bjdv6n1-301

Panza, M. R., Donegá, M. V. B., Pacheco, F. M. P., Nagao, E. O., Hara, F. A. S., Cavalheiro, W. C. S., \& Vendruscolo, J. (2020). Características da paisagem para manejo dos recursos naturais na microbacia do Rio Jacuri, Amazônia Ocidental, Brasil. Brazilian Journal of Development, 6(12), 101532-101558. $10.34117 /$ bjdv6n12-592

Parvis, M. (1950). Drainage pattern significance in airphoto identification of soils and bedrocks. Photogrammetric Engineering, 16, 387-408. 
Pereira, A. S., Shitsuka, D. M., Parreira, F. J., \& Shitsuka, R. (2018). Metodologia da Pesquisa Científica. Universidade Federal de Santa Maria. https://repositorio.ufsm.br/bitstream/handle/1/15824/Lic_Computacao_Metodologia-Pesquisa-Cientifica.pdf?sequence=1

Pittelkow, C. M., Liang, X., Linquist, B. A., Groenigen, K. J. V., Lee, J., Lundy, M. E., Gestel, N. V., Six, J., Venterea, R. T., \& Kessel, C. V. (2014). Productivity limits and potentials of the principles of conservation agriculture. Nature, 517(7534), 365-368. 10.1038/nature13809

Ribeiro, L., Koproski, L. P., Stolle, L., Lingnau, C., Soares, R. V., \& Batista, A. C. (2008). Zoneamento de riscos de incêndios florestais para a Fazenda Experimental do Canguiri, Pinhais (PR). Floresta, 38(3), 561-572. 10.5380/rf.v38i3.12430

Romero, V., Formiga, K. T. M., \& Marcuzzo, F. F. N. (2017). Estudo hidromorfológico de bacia hidrográfica urbana em Goiânia/GO. Ciência e Natura, 39(2), 320-340. 10.5902/2179460X26411

Santos, A. M., Targa, M. S., Batista, G. T., \& Dias, N. W. (2012). Análise morfométrica das sub-bacias hidrográficas Perdizes e Fojo no município de Campos do Jordão, SP, Brasil. Revista Ambiente \& Água, 7(3), 195-211. 10.4136/1980-993X

Santos, R. D., Lemos, R. C., Santos, H. G., Ker, J. C., Anjos, L. H. C., \& Shimizu, S. H. (2013). Manual de descrição e coleta de solo no campo. Viçosa-MG: Sociedade Brasileira de Ciência do Solo.

SEDAM - Secretaria de Estado do Desenvolvimento Ambiental (2002). Atlas Geoambiental de Rondônia. SEDAM.

Silva Neto, A. F., Guimarães, C. L., Araújo, J. S., \& Araújo, J. S. (2013). Geotecnologias para a caracterização morfométrica de bacia hidrográfica. XVI Simpósio Brasileiro de Sensoriamento Remoto. Foz do Iguaçu-PR: Instituto Nacional de Pesquisas Espaciais. p.5483-5490. http://marte2.sid.inpe.br/col/dpi.inpe.br/marte2/2013/05.28.23.12.19/doc/p0267.pdf?ibiurl.language=en

Silva, Q. D. (2012). Mapeamento geomorfológico da Ilha do Maranhão. Presidente Prudente-SP: Universidade Estadual Paulista.

Silva, C. J., Sousa, K. N. S., Ikeda-Castrillon, S. K., Lopes, C. R. A. S., Nunes, J. R. S., Carniello, M. A., Mariotti, P. R., Lazaro, W. L., Morini, A., Zago, B. W., Façanha, C. L., Albernaz-Silveira, R., Loureiro, E., Viana, I. G., Oliveira, R. F., Cruz, W. J. A., Arruda, J. C., Sander, N. L., Freitas Junior, D. S., Pinto, V. R., Lima, A. C., \& Jongman, R.H. G. (2015). Biodiversity and its drivers and pressures of change in the wetlands of the Upper Paraguay-Guaporé Ecotone, Mato Grosso (Brazil). Land Use Policy, 47, 163-178. 10.1016/j.landusepol.2015.04.004

Soares, G. S., Santos Junior, N. R. F., Lima, A. C. R., Bento, A. R., Vendruscolo, J., \& Rosell, E. C. F. (2019a). Caracterização morfométrica da sub-bacia hidrográfica do rio Capitão Cardoso Tenente Marques, Rondônia, Brasil. Revista Geográfica Venezolana, especial, 28-39. http://www.saber.ula.ve/handle/123456789/46153

Soares, G. S., Santos Júnior, N. R. F., Mira, S. F., Moreto, R. F., Cavalheiro, W. C. S., Vendruscolo, J., \& Rosa, D. M. (2019b). Uso de plataforma SIG na caracterização morfométrica da microbacia do rio Santa Teresinha, Amazônia Ocidental, Brasil. Revista Geográfica Venezolana, especial, 84-95. http://www.saber.ula.ve/handle/123456789/46157

Schumm, S. A. (1956). Evolution of drainage systems and slopes in badlands at Perth Amboy, New Jersey. Geological Society of America Bulletin, 67(5), 597646. 10.1130/0016-7606(1956)67[597:EODSAS]2.0.CO;2

Strahler, A. N. (1954). Quantitative geomorphology of erosional landscapes. XIX International Geologic Congress, 13(15), 341-354.

Tambosi, L., Vidal, M. M., Ferraz, S. F. B., \& Metzger, J. P. (2015). Funções eco-hidrológicas das florestas nativas e o Código Florestal. Estudos Avançados, 29(84), 151-162. 10.1590/S0103-40142015000200010

Targa, M. S., Batista, G. T., Diniz, H. D., Dias, N. W., \& Matos, F. C. (2012). Urbanização e escoamento superficial na bacia hidrográfica do Igarapé Tucunduba, Belém, PA, Brasil. Revista Ambiente \& Água, 7(2), 120-142. 10.4136/1980-993X

Teodoro V. L. I., Teixeira D., Costa, D. J. L., \& Fuller, B. B. (2007). O conceito de Bacia Hidrográfica e a importância da caracterização morfométrica para o entendimento da dinâmica ambiental local. Revista UNIARA, 20, 137-157. https://www.uniara.com.br/legado/revistauniara/pdf/20/RevUniara20_11.pdf

Tucci, C. E. M. (1993). Hidrologia: Ciência e Aplicação. (4a ed.), Editora UFRGS.

Vendruscolo, J., Araujo, M. G. S., Ferreira, L. R., Rosa, D. M., \& Hara, F. A. S. (2021). O uso de geotecnologias na caracterização geométrica, topográfica e hidrográfica da microbacia do rio Tamarupá, Amazônia Ocidental, Brasil. Brazilian Journal of Development, 7(1), 4245-4264. 10.34117/bjdv7n1-286

Vendruscolo, J., Pacheco, F. M. P., Ramos, H. F., Cavalheiro, W. C. S., \& Rodrigues, A. A. M. (2020a). Hidrogeomorfometria da microbacia Alto Rio Escondido: informações para auxiliar o manejo dos recursos naturais na Amazônia ocidental. Brazilian Journal of Development, 6(3), 9709-9730. 10.34117/bjdv6n3-011

Vendruscolo, J., Pacheco, F. M. P., Rodrigues, A.A.M., Ramos, H. F., Rosa, D. M., \& Cavalheiro, W. C. S. (2020b). Características morfométricas da microbacia do Médio Rio Escondido, Amazônia Ocidental, Brasil. Brazilian Journal of Development, 6(1), 565-585. 10.34117/bjdv6n1-040

Villela, S. M., \& Mattos, A. (1975). Hidrologia aplicada. McGraw-Hill. 